\title{
Durum wheat genomics comes of age
}

\author{
Introduction to the special issue on durum wheat genomics
}

\author{
Roberto Tuberosa $\cdot$ Curtis Pozniak
}

Published online: 27 November 2014

(C) Springer Science+Business Media Dordrecht 2014

The past two decades have witnessed remarkable progress in developing and deploying plant genomics platforms to identify agronomically valuable loci and, ultimately, enhance crop performance. Accordingly, the articles of this special issue provide a timely update, certainly not exhaustive, on the latest developments in durum wheat genomics and its applications toward the improvement of this valuable crop, a staple food for several hundred million people worldwide. The growing interest in durum wheat is witnessed by a number of international initiatives that focus on this crop and also by the publication earlier this year of another special issue on durum wheat (Able and Atienza 2014) that covers a broad range of disciplines other than genomics and breeding.

Although genome complexity and polyploidy have hindered the development of genomics platforms in both durum and bread wheat, the recent release of high-quality sequence information from hexaploid wheat (Choulet et al. 2014) coupled with the availability of high-density consensus maps for tetraploid

\footnotetext{
R. Tuberosa ( $\bowtie)$

Department of Agricultural Sciences (DipSA), University of Bologna, Viale Fanin 44, 40127 Bologna, Italy e-mail: roberto.tuberosa@unibo.it

C. Pozniak

Department of Plant Science, Crop Development Centre, University of Saskatchewan, 51 Campus Drive,

Saskatoon, SK, Canada
}

wheat (Marone et al. 2012; Maccaferri et al. 2014) has accelerated marker and gene discovery in durum wheat, thus facilitating the genetic dissection of key agronomic traits, as clearly shown by the articles of this special issue, the first one of its kind. Hereafter, we report the highlights of these articles.

Advances in next-generation sequencing have significantly facilitated the discovery of single nucleotide polymorphisms (SNPs) by whole-genome or reducedrepresentation sequencing. However, sequencing in crops with highly repetitive, complex genomes like that of durum wheat can still be challenging and costly. Genotyping-by-sequencing (GBS) can be used for de novo genotyping and SNP discovery, even for the polyploid durum wheat genome. Although several strategies are available for reducing sequencing complexity, David et al. (this issue) focused on sequences of the transcribed portion in 179 lines from a durum wheat pre-breeding population. Using strict filtering, over 103,000 SNPs were successfully identified and revealed that transcription and diversity levels were higher in distal regions of all 14 durum wheat chromosomes. The SNPs and the diversity population from which they were derived will indeed be a useful resource for GWAS or genomic prediction studies.

High-density SNP arrays have been developed for a number of economically important crops, including a $90 \mathrm{~K}$ iSelect array for bread wheat (Wang et al. 2014). Distelfeld et al. (this issue) deployed the $90 \mathrm{~K}$ iSelect array on a highly polymorphic mapping population 
derived from the cross between wild emmer wheat and durum wheat. This high-density map includes 14,088 markers grouped into 2,296 genetic loci uniformly distributed on all linkage groups, corresponding to the 14 chromosomes of tetraploid wheat. Notably, the comparison of this map with the barley map showed synteny for $92.4 \%$ of markers, an encouraging finding for comparative genomics studies and gene discovery. The $90 \mathrm{~K}$ iSelect array was also used to develop highdensity linkage maps to localize QTLs associated with yellow color (Blanco et al. this issue) as well as kernel morphology and thousand-kernel weight (Mastrangelo et al. this issue). In the latter case, the high-density map of 9,040 markers identified six QTLs associated with kernel morphology and thousand-kernel weight. Although the level of polymorphism reported by Blanco et al. (this issue) was only $30 \%$ of that reported by Distelfeld et al. (this issue), their integrated map allowed for the identification of nine QTLs for yellow pigment content, two of which showed consistent effects in the four environments that were tested. The genome scan for QTLs and the SNP-homology prediction against annotated proteins in wheat and Brachypodium genomes identified three candidate genes of the carotenoid biosynthesis pathway which were significantly associated with yellow pigment content, a finding that provides the basis for markerassisted selection (MAS) for yellow pigment color.

Wheat domestication and subsequent evolution under domestication caused significant genetic changes that affected plant morphology, physiology and phenology. In this context, the main goal of Tzarfati et al. (this issue) was the genetic dissection of the key domestication trait (brittle rachis) and traits evolved under domestication, based on quantitative phenotyping of a RIL population derived from a cross between durum and wild emmer wheat. Novel QTLs for rachis fragility, spike threshing and other domestication-related traits were identified and confirmed some of the known putative locations for QTLs affecting these traits. Overall, the number of domestication-related QTLs mapped to the A genome was twofold higher than those found on the B genome, hence supporting the concept of "genome asymmetry." These results contribute to a better understanding of the initial steps of domestication evolution of cereals.

The applicative value of genomics approaches relies on MAS for the desired alleles (Randhawa et al. 2013) at known loci and/or genomic selection based on the use of indices to predict the breeding value of each progeny according to its molecular profile (Storlie and Charmet 2013). While genomic selection remains largely unexplored in durum wheat, numerous studies have reported markers linked to loci that control valuable agronomic traits and ultimately grain yield. Gadaleta et al. (this issue) developed a high-density deletion map of chromosome 5A of tetraploid wheat and identified a major QTL for yield components on the sub-telomeric region. Syntenic analysis of this QTL region with model species revealed the presence of genes involved in basic metabolism and in response to stress, including heatshock proteins, chaperones, serine/threonine protein kinases and membrane transporters. This work will pave the way for cloning of genes important to grain yield in durum wheat.

Root architectural features play a key role in determining yield potential and regulating the adaptive response of wheat to drought and other environmental stresses (Richards 2008). Association mapping allowed Cane' et al. (this issue) to identify QTLs for root system architecture (RSA) in a collection of elite durum wheat genotypes previously evaluated in 15 field environments characterized by a broad range of soil-moisture availability and productivity. The position of QTLs for RSA features was compared with that of QTLs detected for grain yield and other agronomic traits. Among the 48 QTLs detected for RSA, 15 overlapped with QTLs for agronomic traits in two or more environments. In view of its higher heritability and concurrent QTL effects on grain yield, seminal root angle appeared to be the most promising root trait for further studies that investigate the adaptive role of RSA plasticity on field performance in environments differing for water availability/demand.

A key aspect to enhance the effectiveness of genomics-assisted breeding for disease resistance is the identification of QTLs for horizontal (partial) resistance, likely to be more durable as compared to loci for vertical resistance. The genetic analysis of the phenotypic reactions to powdery mildew of the RIL population derived by Ben-David et al. (this issue) from a cross between durum and wild emmer wheat revealed the presence of five independent QTLs for partial resistance to powdery mildew, with the durum parent contributing the resistance allele at a major QTL that explained $26 \%$ of the variance, a remarkable result that emphasizes the high breeding potential of tetraploid wheat germplasm. 
The secondary and tertiary gene pools of durum wheat are a useful source of disease resistance, and tagging these sources with DNA markers will be useful for durum wheat breeders to speed introgression into adapted germplasm. Leaf rust is an important disease in durum wheat (Singh et al. 2013), and under severe epidemics can cause significant reduction in grain yield and quality in most growing regions. Desiderio et al. (this issue) used a high-density map of approximately 11,000 loci to localize three genomic regions associated with resistance to the leaf rust disease from 'MG5423', an accession of emmer wheat (Triticum turgidum ssp. dicoccum). Comparison of QTL positions with those of other previously identified wheat leaf rust resistance genes suggests that those from 'MG5423' are novel resistance sources. Accordingly, the markers tagging these QTLs will indeed be useful to breeders for pyramiding resistance QTL alleles, particularly those showing positive epistatic effects as noted by the authors.

A more challenging and harmful fungal pathogen is Fusarium head blight (FHB), a global threat for durum yield and grain quality due to mycotoxin contamination. Enhancing FHB resistance has proven very difficult due to its low heritability and the narrow genetic variation for this trait in the durum wheat gene pool. Broadening the genetic basis by incorporating resistance alleles from wild and cultivated durum relatives is a promising approach for enhancing durum resistance to FHB. The review by Prat et al. (this issue) summarizes the current information on FHB resistance improvement in durum wheat, including also wild and cultivated tetraploid wheat, hexaploid wheat and alien species. Notably, the QTLs found in tetraploid wheat populations largely overlap with the QTLs identified in hexaploid wheat, suggesting a common genetic basis of FHB resistance. Using a high-density SNP map, Xu et al. (this issue) reported three QTLs for FHB resistance in a population derived from a cross between a domesticated accession of emmer wheat and 'Ben', a highly susceptible cultivar. Resistance QTLs were identified on chromosomes $3 \mathrm{~A}$ and $5 \mathrm{~A}$ from the emmer accession, and on chromosome 2A, with resistance contributed by 'Ben'. Several related Triticeae species have been found to express effective resistance. One strategy to improve FHB resistance in the elite durum wheat gene pool is to introgress resistances by means of chromosome engineering. Forte et al. (this issue) used a cytogenetic strategy to successfully transfer into durum wheat a
QTL allele for FHB resistance from Thinopyrum ponticum. Pyramiding into durum of the target QTL allele from two $T$. ponticum accessions was achieved, and tetraploid lines exhibited a 70-85\% reduction in FHB severity following inoculation with Fusarium and a corresponding decrease in fungal biomass in kernels of infected spikes. These findings support the use of the secondary and tertiary gene pool as a rich reservoir of genes that can be used to improve disease resistance of durum wheat. Based on these findings, FHB resistance breeding by allele introgression into durum wheat is feasible and pyramiding QTLs from related tetraploid species with native durum QTLs will be a viable and valuable strategy for improving FHB resistance in durum wheat.

Overall, the manuscripts of this special issue highlight the value and power of genomics to dissect the genetic basis of traits and the importance of wild germplasm in contributing novel alleles of breeding value at target loci. In view of the low level of polymorphism of elite durum germplasm (Maccaferri et al. 2005), the availability of well-saturated genetic maps is essential for accurate haplotyping of germplasm collections and particularly for fine mapping and positional cloning. We hope that the articles published in this special issue will prove useful to those interested in using genomics approaches in order to improve durum wheat productivity and quality while advancing our understanding of their genetic and functional bases. Additionally, these articles provide a valuable reference collection for the durum research community engaged in gene/QTL discovery in tetraploid wheat. Finally, in view of recent progress in sequencing and assembling large genomes, we hope that it will soon be possible to sequence the durum genome and thus bridge more effectively tetraploid and hexaploid wheat genomics and breeding.

We wish to thank the authors for their valuable contributions, the funding agencies for supporting their work and all the colleagues who have competently reviewed the manuscripts of this special issue.

\section{References}

Able J, Atienza S (2014) Durum wheat for the future: challenges, research and prospects in the 21 st century. Crop Pasture Sci Spec Issue 65(1):124

Choulet F, Alberti A, Theil S, Glover N, Barbe V, Daron J, Pingault L, Sourdille P, Couloux A, Paux E, Leroy P, 
Mangenot S, Guilhot N, Le Gouis J, Balfourier F, Alaux M, Jamilloux V, Poulain J, Durand G, Bellec A, Gaspin C, Safar J, Dolezel J, Rogers J, Vandepoele K, Aury JM, Mayer K, Berges H, Quesneville H, Wincker P, Feuillet C (2014) Structural and functional partitioning of bread wheat chromosome 3B. Science 345:6194. doi:10.1126/ science. 1249721

Maccaferri M, Sanguineti MC, Noli E, Tuberosa R (2005) Population structure and long-range linkage disequilibrium in a durum wheat elite collection. Mol Breed 15:271-289. doi:10.1007/s11032-004-7012-z

Maccaferri M, Ricci A, Salvi S, Milner SG, Noli E, Martelli PL, Casadio R, Akhunov E, Scalabrin S, Vendramin V, Ammar K, Blanco A, Desiderio F, Distelfeld A, Dubcovsky J, Fahima T, Faris J, Korol A, Massi A, Mastrangelo AM, Morgante M, Pozniak C, N'Diaye A, Xu S, Tuberosa R (2014) A high-density, SNP-based consensus map of tetraploid wheat as a bridge to integrate durum and bread wheat genomics and breeding. Plant Biotechnol J. doi:10. 1111/pbi.12288

Marone D, Laido G, Gadaleta A, Colasuonno P, Ficco DB, Giancaspro A, Giove S, Panio G, Russo MA, De Vita P, Cattivelli L, Papa R, Blanco A, Mastrangelo AM (2012) A high-density consensus map of $\mathrm{A}$ and $\mathrm{B}$ wheat genomes. Theor App Gen 125:1619-1638. doi:10.1007/s00122-0121939-y

Randhawa HS, Asif M, Pozniak C, Clarke JM, Graf RJ, Fox SL, Humphreys DG, Knox RE, DePauw RM, Singh AK,
Cuthbert RD, Hucl PJ, Spaner D (2013) Application of molecular markers to wheat breeding in Canada. Plant Breed 132:458-471. doi:10.1111/pbr.12057

Richards RA (2008) Genetic opportunities to improve cereal root systems for dryland agriculture. Plant Prod Sci 11:12-16

Singh A, Pandey MP, Singh AK, Knox RE, Ammar K, Clarke JM, Clarke FR, Singh RP, Pozniak CJ, DePauw RM, Cutherbert RD, Randhawa HS, Fetch TG (2013) Identification and mapping of leaf, stem and stripe rust resistance quantitative trait loci and their interactions in durum wheat. Mol Breed 31:405-418. doi:10.1007/s11032-012-9798-4

Storlie E, Charmet G (2013) Genomic selection accuracy using historical data generated in a wheat breeding program. Plant Genome 6(1). doi: 10.3835/plantgenome2013.01. 0001

Wang S, Wong D, Forrest K, Allen A, Chao S, Huang BE, Maccaferri M, Salvi S, Milner S, Cattivelli L, Mastrangelo A, Whan A, Stephen S, Barker G, Wieseke R, Plieske J, Lillemo M, Mather D, Appels R, Dolferus R, BrownGuedira G, Korol A, Akhunova A, Feuillet C, Salse J, Morgante M, Pozniak C, Luo MC, Dvorak J, Morell M, Dubcovsky J, Ganal M, Tuberosa R, Lawley C, Mikoulitch I, Cavanagh C, Edwards K, Hayden M, Akhunov E (2014) Characterization of polyploid wheat genomic diversity using a high-density 90,000 SNP array. Plant Biotechnol J. doi:10.1111/pbi.12183 